\title{
Relationship between Change in Flow Property and Coalescence of Oil Droplets in Fish Meat Emulsion with Addition of Egg-White
}

\author{
Teruo Nakayama, Hiroko TOMITA and Atsushi OOI \\ Faculty of Bioresources, Mie University, 1515, Kamihama-cho, Tsu, Mie 514, Japan
}

Received May 17, 1995

\begin{abstract}
Fish meat emulsion was prepared from egg-white, very low lipid sardine meat, salad oil, and vinegar. Its flow property was investigated in relation to coalescence. The peak stresses at 0 , about 10 , and $200 \mathrm{~s}^{-1}$ (P1, P2, P3) represented the shape of the flow curve. During the initial 7 days, an increase in $P 1$ and $P 3$ was observed in the conventional emulsion composed of egg-white, salad oil and vinegar. An increase in P1 and P2 was observed in fish meat emulsion and in the fish meat matrix including egg-white and vinegar. The P1 increase was ascribed to the properties of the egg-white. During this storage period of fish meat emulsion, coalescence proceeded slowly. On and after 10 days, the $P 1$ increased in the conventional emulsion, but it decreased in fish meat emulsion and in the fish meat matrix. The P1 decrease was ascribed to the properties of the fish meat. This P1 decrease was accompanied by rapid progress of the coalescence. The coalescence development depended on the change in ovalbumin at the interface and the flow property of the fish meat matrix.
\end{abstract}

Keywords: fish meat emulsion, egg-white, flow property, coalescence, yield stress

Nutritional substances are richly contained in sardine meat. In our previous study (Nakayama et al., 1992), a new fish meat emulsion was produced from sardine meat preparation with very low lipid content. The effect of egg-yolk or egg-white addition on the flow property of the new fish meat emulsion was investigated (Nakayama et al., 1993a). When egg-yolk was used as an emulsifier, the fish meat emulsion was stable in a shear application and also during storage. When egg-white was used as an emulsifier, a shear-unstable emulsion with large yield stress was formed, and the yield stress was further increased during storage.

In this study, the relationship between the coalescence development and the change in yield stress was pursued from the viewpoint of ingredient combination. The emulsion is composed of dispersed and continuous phases. The change in adsorbed ovalbumin around the oil droplets will be related to the flow property of the emulsion and the coalescence of the dispersed oil phase. The continuous phase contained sardine meat and vinegar. The role and effect of the continuous phase were important in relation to the consistency of the fish meat emulsion and the interaction with the emulsifier ovalbumin molecules. Therefore, to clarify the relationship between the coalescence and the flow property, a non-fish conventional emulsion and a non-oil fish meat matrix were investigated for comparison with the fish meat emulsion including all ingredients. A discussion of this study was done to depict the structure of the fish meat emulsion and examine the cause of the instability of the egg-white added to the fish meat emulsion.

\section{Materials and Methods}

Sardine meat preparation with very low lipid content Two new types of sardine meat preparations (Nakayama et al, 1993a; Nonaka, 1990) were prepared in the pilot plant of Taiyo Fishery Co., Ltd., in Nagasaki prefecture, as shown in Fig. 1. Minced meat of fresh sardines was ground with 4 volumes of cold $0.1 \% \mathrm{NaHCO}_{3}$ and $0.1 \% \mathrm{NaCl}$ solution or suspended in the same solution to remove lipid and blood. After the removal of impurities such as bones and skin, the ground meat was dewatered by centrifugation. Without this impurity removal, the suspended meat was dewatered. Ground or suspended meat thus obtained was mixed with sorbitol and subjected to frozen storage at $-40^{\circ} \mathrm{C}$. The sardine meat preparations produced through grinding or suspending processes were designated PMM-A and PMM-B, respectively, as an abbreviation for Purified Minced Meat. The composition of PMM-A and PMM-B was estimated as follows.

PMM-A: moisture, 73.3\%; protein (myofibrillar fraction), 19.6\%; lipid, 1.1\%; ash, 0.8\%; carbohydrate (sorbitol), $5.0 \%$.

PMM-B: moisture, 69.7\%; protein (myofibrillar fraction), 22.5\%; lipid, 1.8\%; ash, 0.8\%; carbohydrate (sorbitol), 5.0\%.

The new type emulsion was prepared from PMM-A or PMM-B as previously reported (Nakayama et al., 1992, 1993a). As an emulsifier, egg-white was used. To adjust the moisture content of the emulsion, the ratio of water added to PMM-A and PMM-B was set at 0.1 and 0.2 , respectively (Nakayama et al, 1994). The constituent ratio of the fish meat emulsion including all ingredients was:

PMM-A or PMM-B : egg-white : salad oil : vinegar $=1: 0.7: 1.1: 0.33$

One or two ingredients were omitted in some cases. The ingredients of each emulsion and its matrix are shown in the figure.

Measurement of flow property A shear rate sweep measurement was carried out with a cone-and-plate 


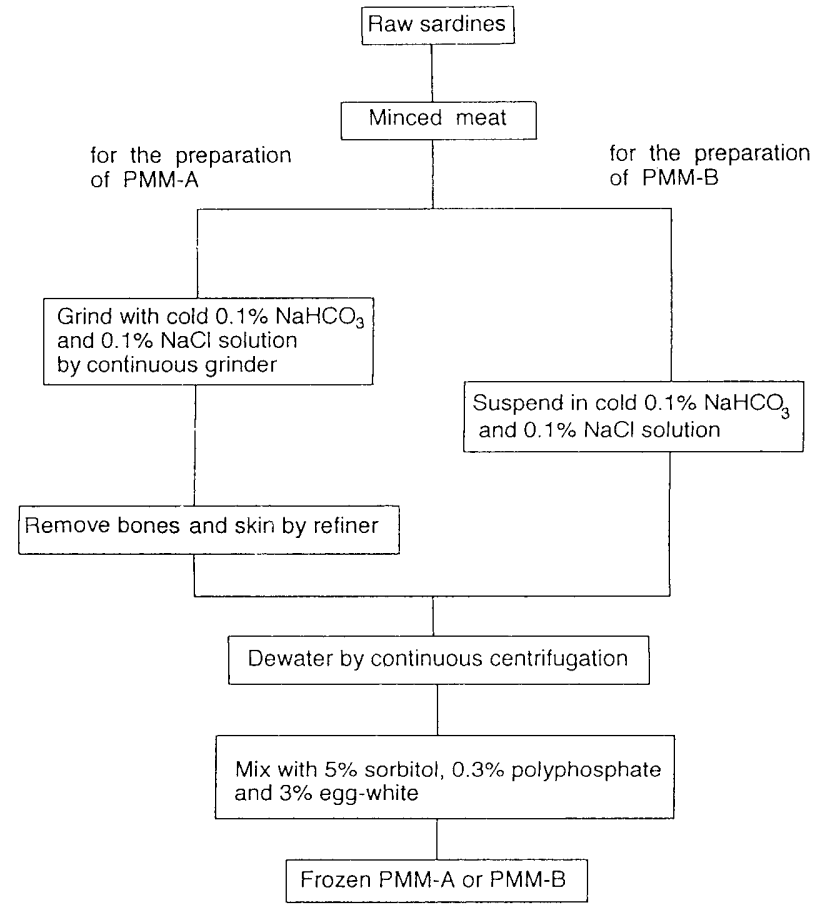

Fig. 1. Outline of procedure for processing Purified Minced Meat. PMM, sardine meat preparation with very low lipid content.

rheometer (NRM-100, Nihon Rheology Kiki Co., Ltd.) using a cone (top platen) having an angle of $3^{\circ}$ and a radius of 3.68 $\mathrm{cm}$. The plate (bottom platen) of the instrument was thermostatted at $5 \pm 0.2^{\circ} \mathrm{C}$. The maximum shear rate was $200 \mathrm{~s}^{-1}$, and the sweep times taken for the increasing shear rate curve (hereinafter called the up-curve) and the decreasing shear rate curve (down-curve) were both $100 \mathrm{~s}$.

The response to the applied shear was evaluated by four factors. Peak 1 (P1) and peak 3 (P3) are the stresses detected at a shear rate of $0 \mathrm{~s}^{-1}$ at the start of shear application and at a maximum shear rate of $200 \mathrm{~s}^{-1}$, respectively. Peak 2 (P2) is the largest stress detected between 0 and $20 \mathrm{~s}^{-1}$ for the upcurve. $\mathrm{P} 1$ and $\mathrm{P} 2$ are the yield stresses required for the onset of flow (refer to the Appendix). The fourth factor $(S)$ is the hysteresis loop area surrounded by the up-curve and down-curve.

The flow property of a system composed of egg-white and vinegar belonged to a liquid-type like water. The cone-andplate rheometer was inapplicable to this system. Therefore, a coaxial cylinder type viscometer (BL adapter, Tokyo Keiki Co., Ltd.) was used for the measurement of viscosity. The ratio of the inner cylinder's radius to that of the outer cylinder was 0.908 . The sample temperature was maintained at $5 \pm$ $0.2^{\circ} \mathrm{C}$ by a thermostatted water bath surrounding the measuring head. The shear rates $6.8,13.6,34.2$, and $68.4 \mathrm{~s}^{-1}$ were applied to the sample for $20 \mathrm{~s}$ each.

Microscopic observation The prepared slides of the new type emulsion were observed under a microscope (OPTIPHOT, Nikon Co., Ltd.) with a $40 \times$ objective and a $10 \times$ ocular. Photographs were taken with an automatic photographing system (MICROFLEX AFX-IIA, Nikon Co., Ltd.).

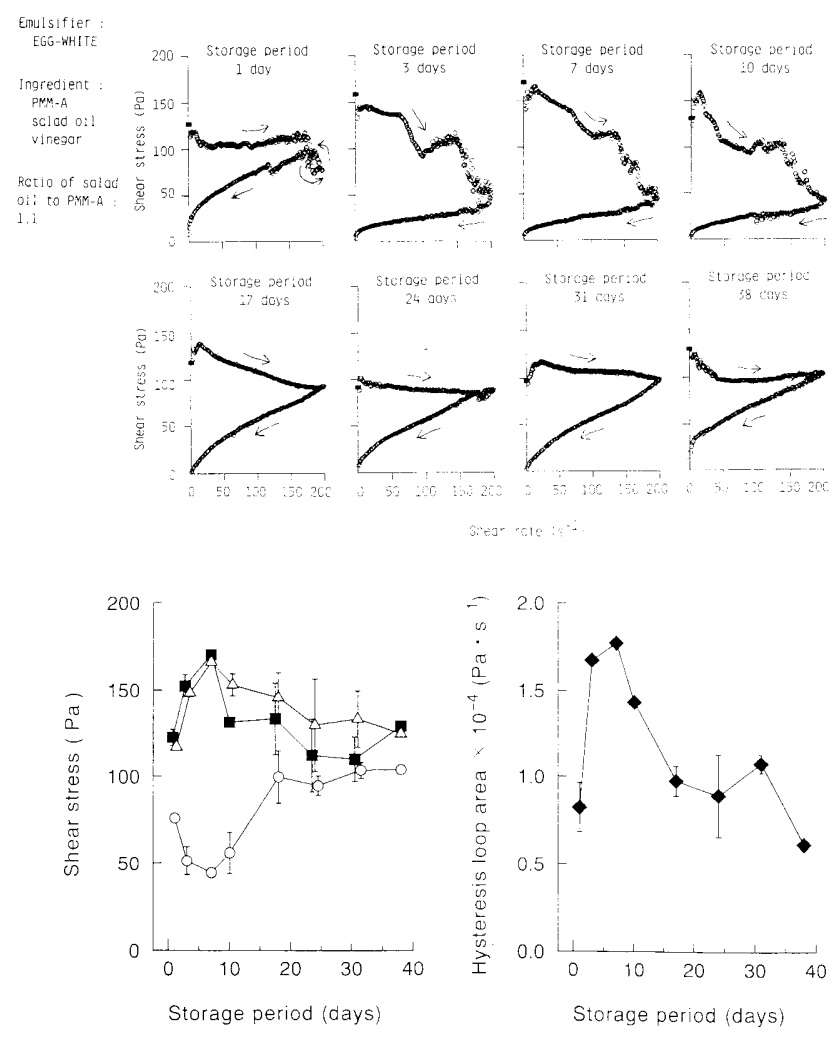

Fig. 2. Flow behavior hysteresis of fish meat emulsion prepared from PMM-A during storage. $\mathbf{m}$, peak 1 (first yield stress) at $0 \mathrm{~s}^{-1} ; \wedge$, peak 2 (second yield stress) at about $10 \mathrm{~s}^{-1}, \cdots$, peak 3 (curve end stress) at $200 \mathrm{~s}^{-1} ; \bullet$, hysteresis loop area.

Mean $\pm \mathrm{SD}, n=6$. The symbols without a vertical bar indicate that the standard deviation bar $(n=6)$ is smaller than the symbol.

\section{Results}

Flow property of fish meat emulsion prepared from PMM-A during storage A typical example of the hysteresis behavior of emulsion including egg-white, PMM-A, salad oil, and vinegar with increasing and decreasing shear rate determination is displayed at the top of Fig. 2. The three peaks, P1, P2, and P3, which represent the shape of each curve are shown at the left bottom and the hysteresis loop area $S$ is shown at the right bottom. The first and second yield stresses at the onset of flow (P1 and P2) increased from 122 to $170 \mathrm{~Pa}$ and from 117 to $166 \mathrm{~Pa}$, respectively during the initial 7 days. Regarding P2, refer to the Appendix. The stress at the maximum shear rate $200 \mathrm{~s}^{-1}(\mathrm{P} 3)$ decreased from 76 to $45 \mathrm{~Pa}$ during this period. Thereafter, $\mathrm{P} 1$ and $\mathrm{P} 2$ decreased and P3 increased. The great difference between the yield stress (P1, $\mathrm{P} 2$ ) and the stress $\mathrm{P} 3$ resulted in the large hysteresis loop area Consequently, the area $S$ increased from 8200 to $17700 \mathrm{~Pa}$. $\mathrm{s}^{-1}$ during the initial 7 days. Thereafter, the $S$ decreased because the difference became small.

The flow behavior hysteresis of a matrix composed of egg-white, PMM-A, and vinegar without a dispersed oil phase is shown at the top of Fig. 3. The three peaks, P1, P2, P3, defined above are shown at the left bottom and the area $S$ at the right bottom. P1, P2, and P3 increased from 73 to 124 $\mathrm{Pa}$, from 82 to $121 \mathrm{~Pa}$, and from 96 to $133 \mathrm{~Pa}$, respectively, during the initial 7 days. Thereafter, these peak values all decreased. The area $S$ increased from 2800 to $4700 \mathrm{~Pa} \cdot \mathrm{s}^{-1}$ 


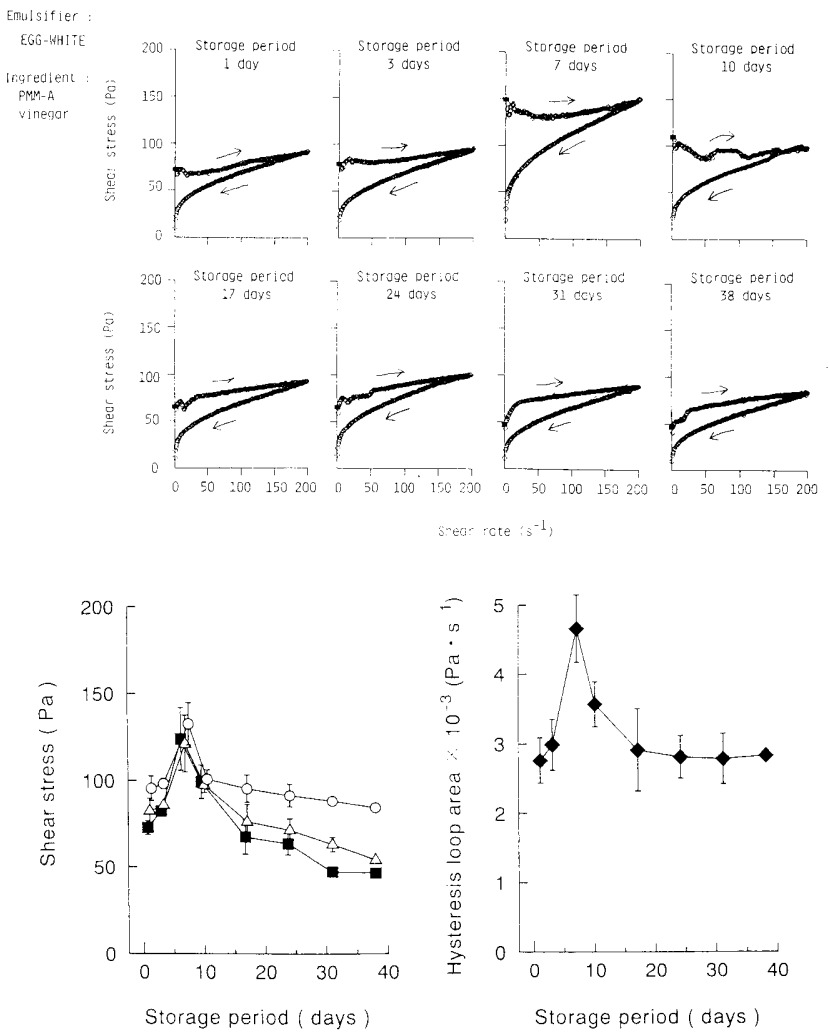

Fig. 3. Flow behavior hysteresis of non-oil fish meat matrix prepared from PMM-A during storage. $\mathbf{a}$, peak 1 at $0 \mathrm{~s}^{-1} ; \triangle$, peak 2 at about $10 \mathrm{~s}^{-1} ; 0$, peak 3 at $200 \mathrm{~s}^{-1} ; \bullet$, hysteresis loop area. Mean $\pm \mathrm{SD}, n=6$. The symbols without a vertical bar indicate that the standard deviation bar $(n=6)$ is smaller than the symbol.

during the initial 7 days, and thereafter decreased.

Flow property of fish meat emulsion prepared from $P M M-B$ during storage The flow behavior hysteresis and the derived parameters of the emulsion including egg-white, PMM-B, salad oil, and vinegar are shown in Fig. 4. The yield stresses, $\mathrm{Pl}$ and $\mathrm{P} 2$, increased from 75 to $161 \mathrm{~Pa}$ and from 95 to $146 \mathrm{~Pa}$, respectively, during the initial 7 days. The stress $\mathrm{P} 3$ decreased from 118 to $67 \mathrm{~Pa}$ during this period. Thereafter, $\mathrm{P} 1$ and $\mathrm{P} 2$ decreased and $\mathrm{P} 3$ increased. At 1 day storage, $\mathrm{P} 1$ and $\mathrm{P} 2$ were smaller than P3. The shape of the hysteresis flow curve was similar to that of the stable fish meat emulsion with the addition of egg-yolk as an emulsifier (Nakayama et al, 1993a, 1993b) and corresponded to the small loop area. The great difference between the yield stress (P1, P2) and the stress P3 for 7 days storage resulted in a large hysteresis loop area. Consequently, the area $S$ increased from 4700 to $7700 \mathrm{~Pa} \cdot \mathrm{s}^{-1}$ during the initial 7 days. Thereafter, $S$ decreased because the difference became small.

The flow behavior and the derived parameters of the matrix composed of egg-white, PMM-B, and vinegar without a dispersed oil phase are shown in Fig. 5. P1, P2, and P3 increased from 40 to $68 \mathrm{~Pa}$, from 42 to $76 \mathrm{~Pa}$, and from 70 to $100 \mathrm{~Pa}$, respectively, during the initial 7 days. Thereafter, these peak values all decreased. The area $S$ increased from 1500 to $3200 \mathrm{~Pa} \cdot \mathrm{s}^{-1}$ during the initial 7 days and thereafter decreased.

Flow property of conventional emulsion composed of egg-white, salad oil, and vinegar during storage The flow behavior hysteresis and the derived parameters of the conven-

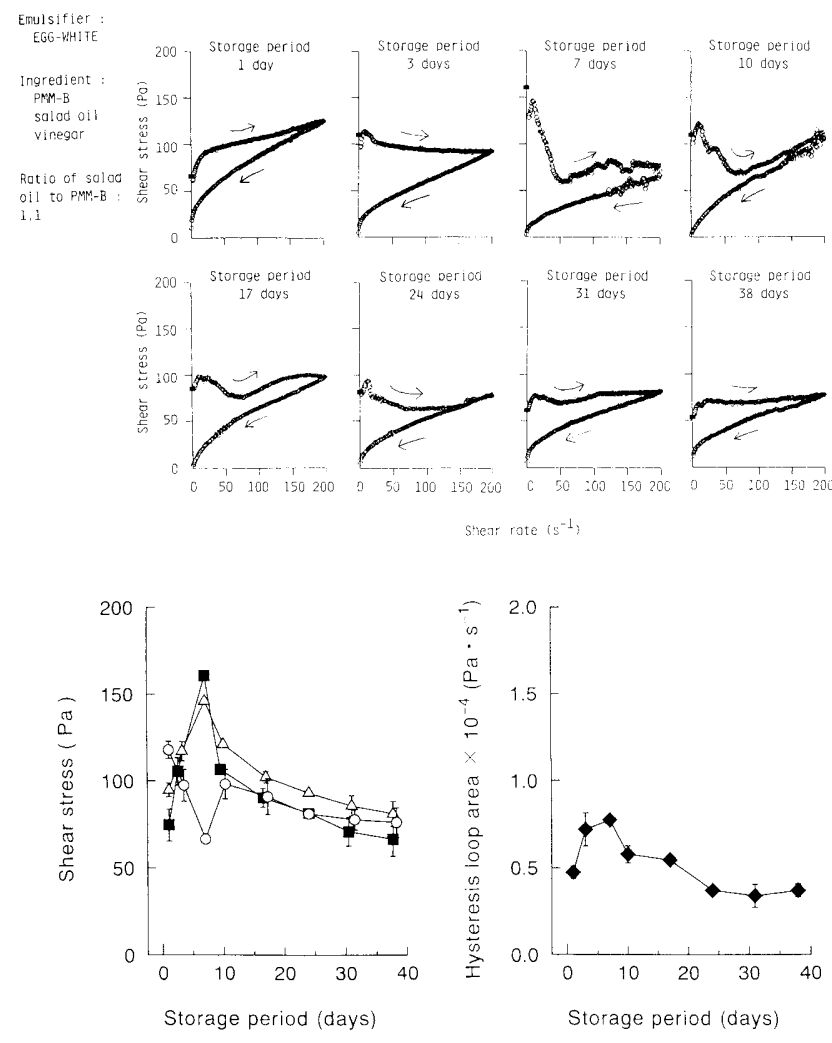

Fig. 4. Flow behavior hysteresis of fish meat emulsion prepared from PMM-B during storage. $\boldsymbol{a}$, peak 1 at $0 \mathrm{~s}^{-1} ; \triangle$, peak 2 at about $10 \mathrm{~s}^{-1} ; 0$, peak 3 at $200 \mathrm{~s}^{-1} ;$, hysteresis loop area. Mean $\pm \mathrm{SD}, n=6$. The symbols without a vertical bar indicate that the standard deviation $\operatorname{bar}(n=6)$ is smaller than the symbol.

tional emulsion including egg-white, salad oil, and vinegar are shown in Fig. 6. The second yield stress $\mathrm{P} 2$ always found in fish meat emulsion was not detected at any storage period except 24 and 31 days. The shape of the hysteresis flow curve was similar to that of the stable fish meat emulsion with the addition of egg-yolk (Nakayama et al, 1993a, 1993b) and corresponded to the small loop area. The conventional emulsion prepared in this study exhibited a small P1 and P3 throughout the storage. The yield stress P1 increased steeply from 7 to $17 \mathrm{~Pa}$ during the initial 7 days, then gradually to 24 $\mathrm{Pa}$ at 24 and 31 days storage and decreased slightly to $19 \mathrm{~Pa}$ at 38 days storage. P3 increased steeply from 22 to $31 \mathrm{~Pa}$ during the initial 7 days storage and thereafter gradually to 38 $\mathrm{Pa}$ at 38 days storage. The area $S$ increased from 400 to 1700 $\mathrm{Pa} \cdot \mathrm{s}^{-1}$ during 31 days storage and decreased slightly to 1300 $\mathrm{Pa} \cdot \mathrm{s}^{-1}$ at 38 days storage. Throughout all the storage periods of the conventional emulsion, $S$ was small even when compared with that of the PMM-B-based matrix.

The sharp increase in $\mathrm{P} 1$ and $\mathrm{P} 3$ of the conventional emulsion during the initial 7 days storage suggested that one of the causes for the $\mathrm{Pl}$ increase in fish meat emulsion during the initial 7 days was ascribed to the egg-white used as an emulsifier. Neither in the system composed of egg-yolk, salad oil, and vinegar nor in that composed of egg-yolk, PMM-A or PMM-B, and vinegar nor in another one composed of egg-yolk, PMM-A or PMM-B, salad oil, and vinegar was the increase in yield stress $\mathrm{P} 1$ during the 38 days storage detected. Therefore, the increase in P1 during storage is a characteristic 


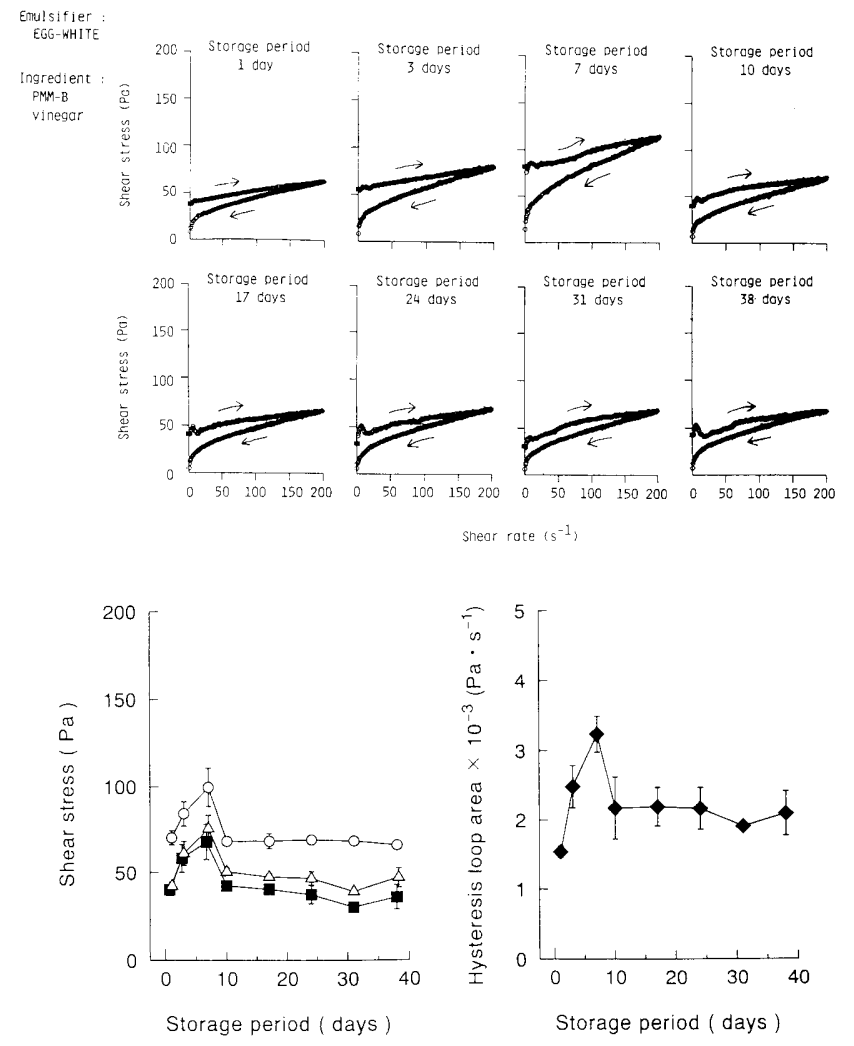

Fig. 5. Flow behavior hysteresis of non-oil fish meat matrix prepared from PMM-B during storage. a, peak 1 at $0 \mathrm{~s}^{-1} ; \triangle$, peak 2 at about $10 \mathrm{~s}^{-1}$; $\bigcirc$, peak 3 at $200 \mathrm{~s}^{-1} ; \bullet$, hysteresis loop area. Mean \pm SD, $n=6$. The symbols without a vertical bar indicate that the standard deviation bar $(n=6)$ is smaller than the symbol.

found only in emulsions containing egg-white.

On and after the 10-day storage, $\mathrm{Pl}$ persistently increased at a slow rate in the conventional emulsion, but it decreased in the fish meat emulsion and also in the fish meat matrix. The persistent increase in $\mathrm{Pl}$ of the conventional emulsion did not agree with the decrease in $\mathrm{Pl}$ of fish meat emulsion in this period. Therefore, the P1 decrease was ascribed to the property of the fish meat.

Flow property of system composed of egg-white and vinegar This system exhibited Newtonian viscosity independent of the shear rate between 6.8 and $68.4 \mathrm{~s}^{-1}$ used in the present experiment. The viscosity was $2.7 \mathrm{mPa} \cdot \mathrm{s}$ and remained almost unchanged during the storage, as shown in Fig. 7. The $\mathrm{pH}$ of the system was originally 4.25 . For comparison with the fish meat emulsion ( $\mathrm{pH} 4.90$ ), a viscosity measurement was carried out also using the sample whose $\mathrm{pH}$ was adjusted to 4.90 . This sample exhibited a viscosity of 2.7 $\mathrm{mPa} \cdot \mathrm{s}$, and the value was unchanged during the storage.

Microscopic observation of coalescence development during storage A microscopic view of the coalescence in fish meat emulsion is shown in Fig. 8. The left row displays the change in the PMM-A-based emulsion during storage; the right row is provided for the PMM-B-based emulsion. During the initial 7 days, the coalescence of oil droplets proceeded slowly in both emulsions. On and after the 10 days storage, rapid progress of the coalescence occurred. A microscopic observation of the conventional emulsion is shown in Fig. 9. During the initial 7 days, coalescence proceeded slowly and

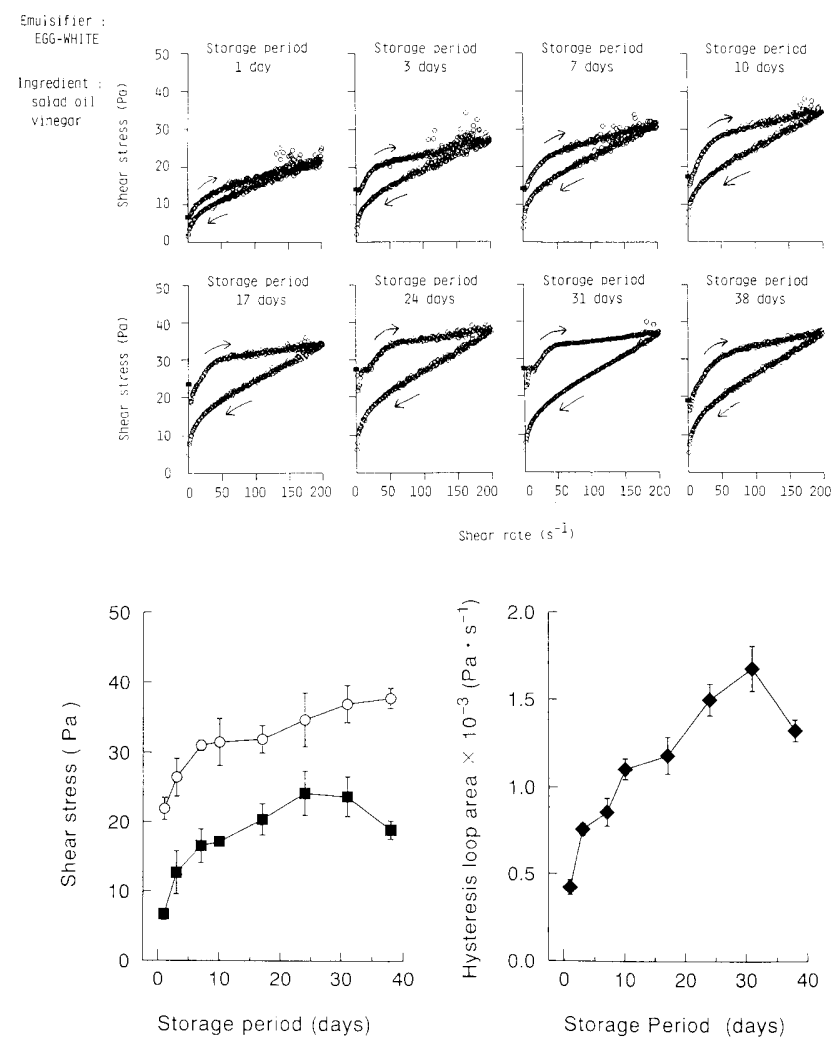

Fig. 6. Flow behavior hysteresis of non-fish conventional emulsion with addition of egg-white as an emulsifier during storage. $\mathbf{a}$, peak 1 at $0 \mathrm{~s}^{-1} ; \triangle$, peak 2 at about $10 \mathrm{~s}^{-1} ; 0$, peak 3 at $200 \mathrm{~s}^{-1}$; $\diamond$, hysteresis loop area. Mean $\mathrm{SD}, n=6$. The symbols without a vertical bar indicate that the standard deviation bar $(n=6)$ is smaller than the symbol.

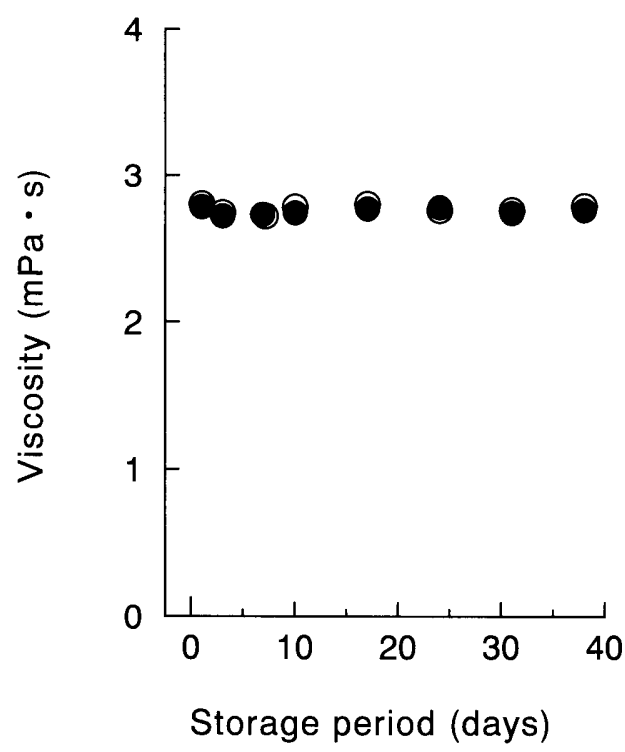

Fig. 7. Flow property of a system composed of egg-white and vinegar during storage., $\mathrm{pH} 4.25 ; \mathrm{pH} 4.90$. The same viscosity value was obtained for the application of shear rates $6.8,13.6,34.2$, and $68.4 \mathrm{~s}^{-1}$ for $20 \mathrm{~s}$ each. The value at $34.2 \mathrm{~s}^{-1}$ is plotted in this figure. The average of 6 determinations is shown and the standard deviation is smaller than the symbol at all the points.

thereafter coalescence development ceased.

The percentages of salad oil relative to the total amount of constituents except for egg-white were 43.5 and $41.8 \%$ in PMM-A and PMM-B-based emulsions, while it was $76.9 \%$ in 
Emulsifier : EGG-WHITE

Ingredient: $P M M-A$, salad oil, vinegar

Ratio of salad oil to PMM-A : 1.1

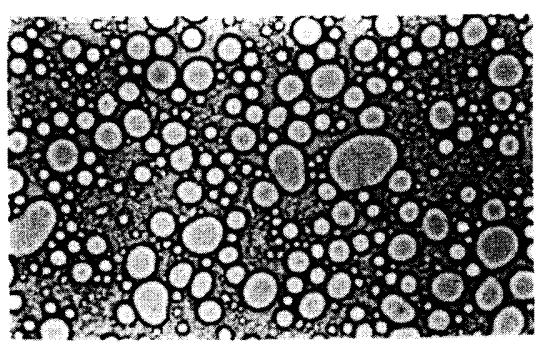

1 d a $y$

$10 \overline{\mu m}$

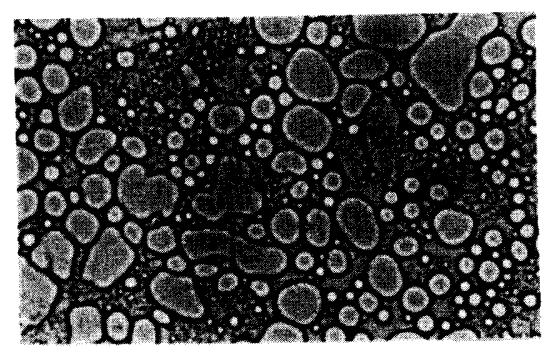

$3 \mathrm{~d}$ a y s $\quad 10 \overline{\mu m}$

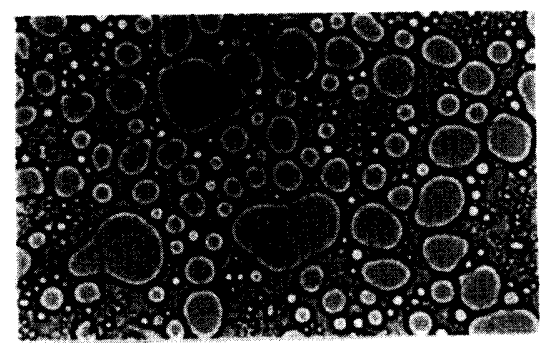

7 d a y s

$10 \overline{\mu \mathrm{m}}$

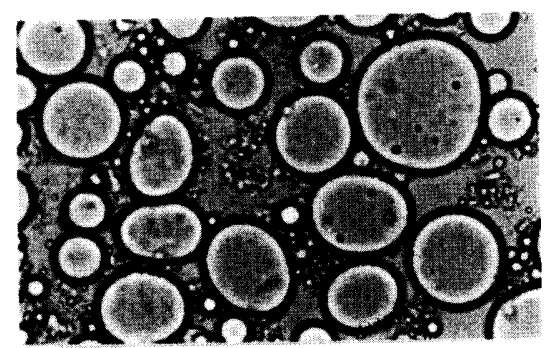

10 d a y s

$10 \overline{\mu m}$

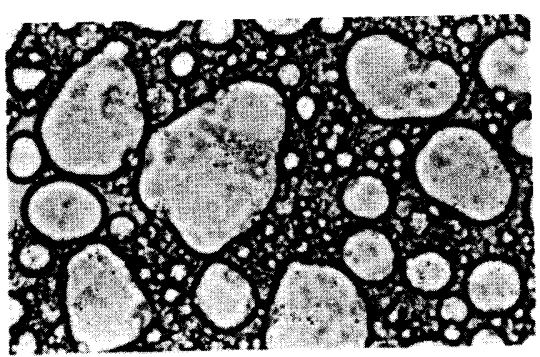

17 d a y s

$10 \bar{\mu} \mathrm{m}$

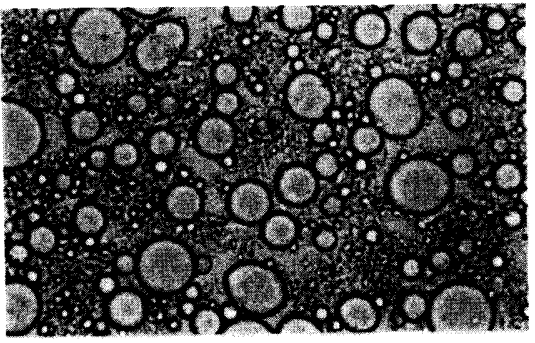

7 d a y s

$10 \overline{\mu \mathrm{m}}$

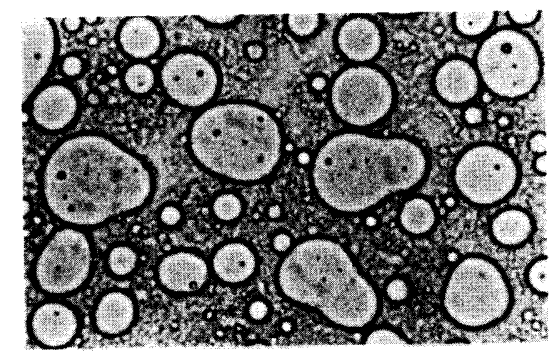

10 d a y s

$10 \overline{\mu m}$

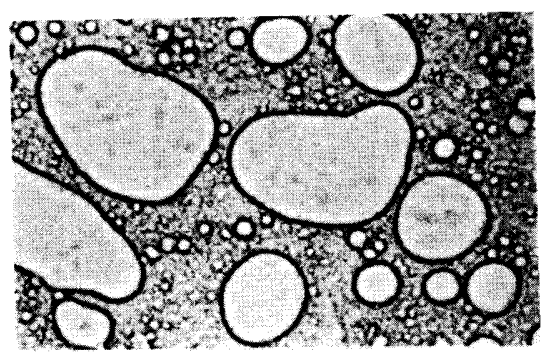

17 d a y s

$10 \overline{\mu m}$

Fig. 8. Coalescence development in fish meat emulsion during storage observed by microscopy.
Emulsifier : EGG-WHITE

Ingredient: $P M M-B$, salad oil, vinegar

Ratio of salad oil to PMM-B: 1.6

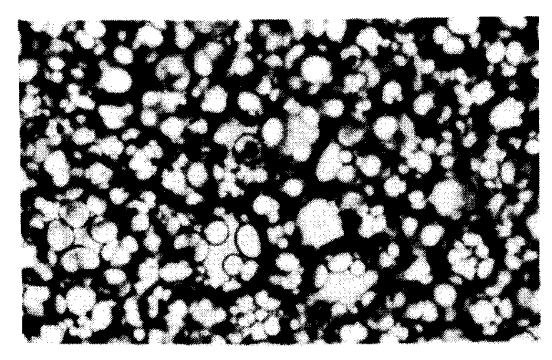

$1 \mathrm{~d}$ a $y$

$10 \mu \mathrm{m}$

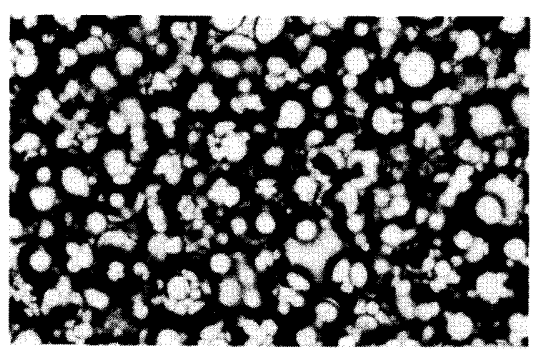

3 d a y s

$10 \overline{\mu \mathrm{m}}$

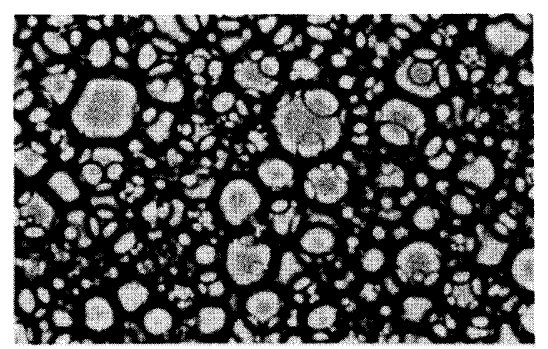

7 d a y s

$10 \overline{\mathrm{m}}$

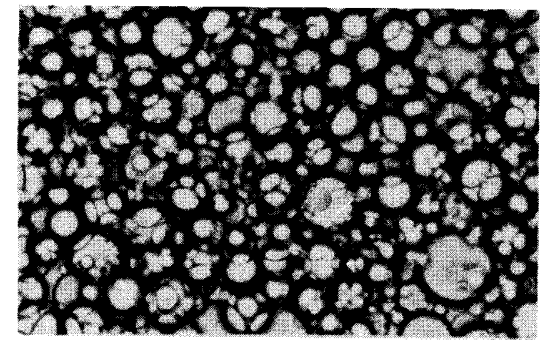

10 d a y s

$10 \overline{\mu m}$

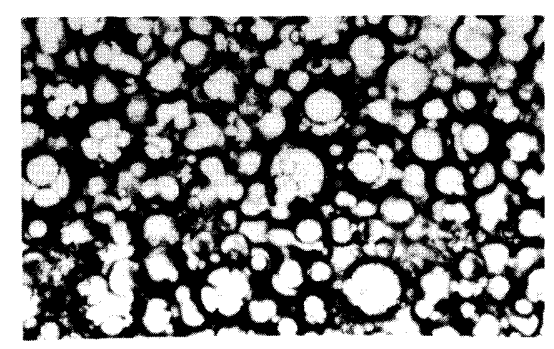

17 d a y s

$10 \overline{\mu m}$

Fig. 9. Coalescence development in non-fish conventional emulsion during storage observed by microscopy. 
the conventional emulsion. The oil percentage of the conventional emulsion corresponded to the oil content of closest packing (Lowe, 1958; Romanoff \& Romanoff, 1949). Therefore, an overlap of oil droplets was seen all over the photograph of this emulsion. We tried to prepare thinner slides for microscopic observation, but our trial resulted in failure because deformation of the oil droplets and separation of the oil phase were induced. A similar overlapping view of the emulsion with the addition of egg white was reported by Miyaguchi et al. (1992).

As the loop area increased in the flow curve of PMM-Abased emulsion, the oil droplet size after flow property measurement became large. Before and after the flow measurement, the modal diameter (droplet size most frequently seen : Arakawa, 1977) was 6.3 and $11.7 \mu \mathrm{m}$ at 1 day storage, 10.0 and $41.7 \mu \mathrm{m}$ at 7 days storage, 34.4 and $41.7 \mu \mathrm{m}$ at 17 days storage, respectively.

For the PMM-B-based emulsion, an increase in the loop area resulted in the large droplet size detected after the flow measurement. Before and after the flow measurement, the modal diameter was 3.8 and $6.7 \mu \mathrm{m}$ at 1 day storage, 6.3 and $20.0 \mu \mathrm{m}$ at 7 days storage, 28.1 and $33.3 \mu \mathrm{m}$ at 17 days storage, respectively. Both the loop area and the droplet size of the PMM-B-based emulsion before and after the flow measurement were smaller than those of the PMM-A-based one.

In a non-fish conventional emulsion, the modal diameter of a droplet was similar before and after the flow measurement. It was 4.7 and $5.0 \mu \mathrm{m}$ at 1 day storage, 7.8 and $9.2 \mu \mathrm{m}$ at 7 days storage, 7.8 and $10.0 \mu \mathrm{m}$ at 17 days storage, respectively. The diameter increased up to 7 days storage and thereafter remained almost unchanged.

\section{Discussion}

In this section, we tried to discuss the relationship of all ingredients in fish emulsion, and the question of whether a single ingredient or two induced the initial consistency change in fish meat emulsion during storage was considered to be solved. We investigated all possible instances of ingredient combination as follows.

System 1: egg-white, vinegar

System 2: egg-white, salad oil, vinegar

System 3: egg-white, PMM-A or PMM-B, vinegar

System 4: PMM-A or PMM-B, salad oil, vinegar

System 5: egg-white, PMM-A or PMM-B, salad oil, vinegar

The isoelectric $\mathrm{pH}$ of ovalbumin, the major protein in egg-white, is 4.5 (Powrie \& Nakai, 1985). The pHs of systems 2,3 , and 5 containing egg-white prepared in this study were all 4.90 (that of system 2 was adjusted by diluting the vinegar with distilled water before mixing with egg-white and salad oil). The $\mathrm{pH}$ of system 1 was originally 4.25 and was adjusted to 4.90 .

System 1 was a non-fish non-oil system, and its flow property was classified into a liquid-type like water. During storage, this property remained almost unchanged, though a very small amount of a flocculent substance was produced. The combination of egg-white and vinegar alone did not become as pasty as mayonnaise. Ovalbumin near the isoelectric point $(\mathrm{pI})$ was dissolved in vinegar but the distance between the ovalbumin molecules was not very short. Interac- tion between ovalbumin molecules did not occur during the storage.

System 2 was a non-fish conventional emulsion. The participation of the dispersed oil brought about the pasty flow property of a mayonnaise type. The percentage of salad oil relative to the total amount of constituents except for egg-white was $76.9 \%$. The oil percentage of this conventional emulsion corresponded to the oil content of closest packing (Lowe, 1958; Romanoff \& Romanoff, 1949). The thick consistency of system 2 was ascribed to the dense condition of closest packing. During storage, a persistent increase in yield stress P1 was detected. Dissolved ovalbumins could migrate to the interface and orient at the surface of the oil droplets with their polar groups directed toward the aqueous phase and their hydrophobic groups toward the oil phase (Kato \& Nakai, 1980). Near the pl, ovalbumins adopt compact structures with high viscoelasticity, due to the lack of repulsive forces. This may stabilize an already adsorbed ovalbumin film around the oil droplet against surface deformation or desorption. However, the distance between ovalbumin molecules was very short in the closest packing condition, and the ovalbumin molecules could come close without the electrostatic repulsion. Therefore, this protein thus adsorbed at the interface would be denatured, and the interaction between the ovalbumin molecules was induced. The increase in P1 and the gradual progress of coalescence during the initial 7 days reflected these changes.

System 3 was a non-oil but fish meat-based system, and during the initial 7 days storage, an increase in P1 was detected. As described in the Results section, the system composed of egg-yolk, PMM-A or PMM-B, and vinegar did not exhibit an increase in Pl during the storage. Therefore, PMM-A and PMM-B did not originally possess a thickening nature during the storage. When PMM-A or PMM-B was included together with egg-white in the system, the property of the Pl increase which was inherent in the conventional emulsion with the addition of egg-white appeared again and became conspicuous. The $\mathrm{pI}$ of fish meat protein, actomyosin, is between 5.0 and 5.5 (Hultin, 1985). Near the pl, the myofibrils composed of actmyosin molecules could come close to ovalbumin molecules without electrostatic repulsion. The interaction between myofibrils and ovalbumin molecules near the $\mathrm{pl}$ intensified the tendency of the P1 increase during the initial 7 days.

System 4 was an emulsified system without the addition of egg-white, and during the preparation, the added oil ratio 0.9 to PMM-A or PMM-B resulted in oil phase separation. The myofibrils which composed PMM-A or PMM-B did not have a good emulsion capacity like that of egg-white.

System 5 was a fish meat emulsion emulsified with eggwhite, and during the initial 7 days storage an increase in $\mathrm{P} 1$ was detected. Ovalbumin adsorbed at the surface of an oil droplet would be denatured during the initial 7 days and gradual progress of coalescence was induced. Both the interaction between ovalbumin molecules (refer to system 2) and the interaction between myofibrils and ovalbumin molecules (refer to system 3 ) would be induced. As the result, the tendency of the P1 increase was intensified much more during the initial 7 days than in system 3 . 
The fish meat emulsion (system 5) developed a yield stress approximately twice that of the non-oil matrix (system 3 ). The presence of salad oil related to the thick consistency of fish meat emulsion. The fish meat emulsion developed a yield stress four times or more as large as that of the non-fish conventional emulsion (system 2). The fish meat caused the thick consistency of the fish meat emulsion as its matrix.

When the fish meat matrix possessed a high viscosity with a P1 increase (systems 3 and 5), the droplets did not easily collide with each other and the coalescence proceeded only slowly. As soon as the matrix exhibited a relatively low viscosity after 10 days storage, the droplets could come close and coalescence proceeded rapidly with the change in adsorbed ovalbumin around the droplets. Therefore, it is concluded that the coalescence development depended on the change in the emulsifier layer and also the consistency of the continuous phase.

As the loop area increased in the flow curve of fish meat emulsions, the oil droplet size after flow property measurement became large. When the matrix structure was weakened by the shear application and the emulsion exhibited a low viscosity in the down-curve of the flow measurement, coalescence proceeded. This phenomenon fell in the same category as the above-described coalescence development.

Nonaka et al. (1989) reported that the PMM-A which was prepared by the grinding with the solution contained myofibrils shorter than $125 \mu \mathrm{m}$ at $60 \%$ of all the myofibrils, and the PMM-B which was prepared by the suspending in the solution contained those longer than $1000 \mu \mathrm{m}$ at $80 \%$. In the present work, the flow property of fish meat emulsion and fish meat matrix during the storage reflected the size of the pulverized myofibrils. Both the change in flow property and the progress of coalescence during storage were more remarkable in the emulsion prepared from PMM-A containing short myofibrils.

Because the myofibrils are pulverized to a shorter size in PMM-A-based samples, the chance of interaction between myofibrils and ovalbumin molecules will increase and this interaction will accelerate the ovalbumin denaturation during cold storage. Probably an aggregate of myofibrils and denatured ovalbumin molecules will be formed during storage much more in PMM-A-based samples than in PMM-B-based ones. As a result, a remarkable change in flow property occurred in both the matrix and the emulsion prepared from PMM-A, and coalescence proceeded faster in the PMM-A-based emulsion due to greater progress of ovalbumin denaturation.

The increase in P1 was accompanied by an increase in P2 during the initial 7 days storage of fish meat emulsion and its matrix. The yield stress P1 would be induced by the breakdown of the primary scaffold structure made of myofibrils and ovalbumin molecules at the onset of shear application. P2 would be induced by the flowing orientation of fragmented myofibrils and oil droplets from random direction arrangement because the fish meat emulsion and its matrix possessed a firm structure with a pasty consistency.

\section{References}

Arakawa, M. (1977). Electron microscopic observation of particle size. In "Lecture on New Experimental Chemistry Vol. 18 Interface and Colloid," 1st Ed., ed. by Chemical Society of Japan. Maruzen, Tokyo, p. 383

Davis, S.S., Khanderia, M.S., Adams, I., Colley, I.R., Cammack, J. and Sanford, P. (1977). Effect of gamma radiation on rheological properties of pharmaceutical semisolids. J. Text. Stud. 8, 61-80.

Hultin, H.O. (1985). Characteristics of muscle tissue. In "Food Chemistry", 2nd Ed., ed. by O.R. Fennema. Marcel Dekker, New York, p. 774.

Kato, A. and Nakai, S. (1980). Hydrophobicity determined by a fluorescence probe method and its correlation with surface properties of proteins. Biochim. Biophys. Acta, 624, 13-20.

Lowe, B. (1958). Experimental Cookery, 4th Ed., John Wiley \& Sons, New York, pp. 278-294.

Miyaguchi, Y., Yuki, S., Nakamura, T. and Tsutsumi, M. (1992). Emulsifying properties of Globin. Nippon Shokuhin Kogyo Gakkaishi, 39, 363-368 (in Japanese).

Nakagawa, T. and Kanbe, H. (1959). Anomalous viscosity. In "Rheology," lst Ed., Misuzu Shobou, Tokyo, pp. 635-639.

Nakayama, T., Oka, T., Ooi, A., Nonaka, M., Hirata, F. and Saeki, H (1992). New emulsified product from sardine meat preparation with very low lipid content. Nippon Shokuhin Kogyo Gakkaishi, 39, 451-456 (in Japanese).

Nakayama, T., Tozaki, Y., Wakabayashi, A. and Ooi, A. (1993a) Effect of yolk and white on shear-stable emulsion preparation from fish. Nippon Shokuhin Kogyo Gakkaishi, 40, 194-204.

Nakayama, T., Wakabayashi, A., Tozaki, Y. and Ooi, A. (1993b). Different effects of whole egg and egg-yolk on emulsion preparation from a fish meat product. Nippon Shokuhin Kogyo Gakkaishi, 40, $872-880$.

Nakayama, T., Tomita, H., Murase, M. and Ooi, A. (1994). Effect of added water amount and yolk white ratio on flow property of fish emulsion. Presented at the 41 th Annual Conference of of the Japanese Society for Food Science and Technology, Tokyo, March $28-30$, p. 128

Nonaka, M., Hirata, F., Saeki, H. and Sasamoto, Y. (1989). Manufacture of highly nutritional fish meat for food stuff from sardines. Nippon Suisan Gakkaishi, 55, 1575-1581 (in Japanese).

Nonaka, M. (1990). Pulverization in water. In "The Nutritive Components of Fish and New Fish Utilization Technology," 1st Ed., ed. by M. Takeuchi. Kouseisha-Kouseikaku, Tokyo, pp. 88-95.

Powrie, W.D. and Nakai, S. (1985). Characteristics of edible fluids of animal origin: eggs. In "Food Chemistry," 2nd Ed., ed. by O.R. Fennema. Marcel Dekker, New York, p. 835.

Romanoff, A.L. and Romanoff, A.J. (1949). "The Avian Egg," Ist Ed. John Wiley \& Sons, New York, pp. 776-778.

\section{Appendix}

Egg-white-containing fish meat emulsion possessed a firm structure in the pasty state. The onset of flow was required to break down the scaffold structure (Nakagawa \& Kanbe, 1959) at first. The appearance of the first yield stress at a shear rate of $0 \mathrm{~s}^{-1}$ reflected this initial breakdown. When the shear rate was gradually increased, a second yield stress was detected between 0 and $20 \mathrm{~s}^{-1}$ (usually at approximately 10 $\mathrm{s}^{-1}$ ). This yield stress was induced by the orientation (Nakagawa \& Kanbe, 1959) of the fish meat matrix and the dispersed oil phase to start the flow. The structure of the fish meat emulsion was brittle. When the shear rate was further increased, the shear stress did not increase any further in some cases and it increased only slightly in the other cases because of the gradual structure breakdown. This type of flow behavior was also reported in pharmaceutical research (Davis et al., 1977). 